\title{
ADAR I may be involved in the proliferation of acute myeloid leukemia cells via regulation of the Wnt pathway
}

This article was published in the following Dove Press journal: Cancer Management and Research

\author{
Han Xiao \\ Qian Cheng \\ Xinyu Wu \\ Yishu Tang \\ Jing Liu \\ Xin $\mathrm{Li}$
}

Department of Hematology, The Third Xiangya Hospital, Central South University, Changsha, Hunan, People's Republic of China
Correspondence: Xin Li; Jing Liu Department of Hematology, The Third Xiangya Hospital, Central South University, Changsha, Hunan, People's Republic of China

Tel/fax +86 7318 86I 80I4

Email lixiner1975@।63.com; lj0jingliu@163.com
Purpose: Acute myeloid leukemia (AML) is the most common type of leukemia and characterized by the malignant growth of leukemic cells. Adenosine deaminases acting on RNA 1 (ADAR1) have been shown to participate in the proliferation of cancer cells and progression of various cancers. However, the role of ADAR1 in AML has not been investigated.

Patients and methods: We compared the expression levels of ADAR1 between samples obtained from different AML patients and controls using quantitative-polymerase chain reaction and Western blotting. We also investigated the functional role and possible mechanisms via silencing the expression of ADAR1 in vitro and in vivo.

Results: We found that the mRNA and protein levels of ADAR1 were significantly higher in AML patients. The mRNA expression of ADAR1 was positively correlated with the ratio of leukemic cells. Additionally, silencing of ADAR1 expression significantly suppressed the proliferation of AML cells and induced G0/1 arrest. For the analysis of the mechanism, the quantitativepolymerase chain reaction and Western blotting results revealed that ADAR1 knockdown resulted in the decreased expression of Wingless-Int (Wnt) effectors including $\beta$-catenin, c-Myc, transcription factor 4, and cyclin D2. In the nude mouse model, inhibition of ADAR1 expression reduced the tumorigenic potential and decreased the expression o]f Wnt effectors.

Conclusion: These results demonstrate that ADAR1 may be involved in the regulation of the proliferation of AML cells partially via regulation of the Wnt signaling pathway.

Keywords: acute myeloid leukemia, ADAR1, proliferation, cell cycle, Wnt pathway

\section{Introduction}

Acute myeloid leukemia (AML) is the most common type of leukemia, with an annual incidence of 3-4/100,000 adults. ${ }^{1} \mathrm{AML}$ is characterized by the uncontrolled proliferation and accumulation of leukemic cells in the bone marrow. ${ }^{2}$ It is generally recognized that the rapid growth of leukemic cells and the deregulation of signaling pathways leads to the development and progression of AML. ${ }^{3}$ Although substantial advancements in improving therapy against AML have been achieved in the previous decades, the prognosis remains poor and the mechanisms of leukemogenesis are not completely understood. ${ }^{4}$ An increasing number of studies have revealed that AML displays a complex variety of genetic changes, which results in the malignant proliferation of AML cells and variable clinical prognosis of AML patients. ${ }^{5}$ Consequently, better understanding of these changes is essential for the effective treatment of AML and design of novel personalized therapies. ${ }^{6}$ 
Adenosine deaminase acting on RNA 1 (ADAR1), a double-stranded RNA-editing enzyme, participates in various physiological and pathological processes. ${ }^{7-10}$ Emerging evidence has shown that ADAR1 is involved in modulating the proliferation, progression, and drug resistance of several cancers including liver, ${ }^{11}$ lung, ${ }^{12}$ and cervical cancer. ${ }^{13}$ It has been demonstrated that the role of ADAR1 in various cancers may be completely distinct. A study reported that overexpression of ADAR1 promoted the growth of lung cancer cells. ${ }^{12}$ In contrast, another study demonstrated that downregulation of ADAR1 may increase the proliferation of metastatic melanoma cells. ${ }^{14}$ In hematological malignancies, it was previously revealed that ADAR1 is essential for the maintenance of both fetal and adult hematopoietic stem cells. ${ }^{10}$ The expression and activity of ADARs are modulated during the differentiation of myeloid cells. ${ }^{15}$ In addition, a formal study showed that ADAR1 was highly expressed in pediatric acute leukemia. ${ }^{16}$ Based on the evidence provided in previous reports, ADAR1 may be a novel diagnostic biomarker and prognostic predictor in leukemia. However, its functions and underlying mechanisms in adult AML have not been fully elucidated.

In this study, we aimed to detect the expression levels of ADAR1 in bone marrow samples obtained from adult patients with AML of different disease status and controls. Furthermore, the roles of ADAR1 were investigated in vivo and in vitro by silencing its expression. Subsequently, several mechanisms potentially involved in regulating the proliferation of AML cells through ADAR1 were also studied.

\section{Materials and methods}

\section{Patients}

All patients included in this study provided written informed consent. The study was approved by the Ethics Committee of The Third Xiangya Hospital of Central South University (Changsha, China). We divided all the newly diagnosed patients or patients in progression into AML $(\mathrm{N}=63)$ and AML-CR groups $(\mathrm{N}=38)$ according to the latest National Comprehensive Cancer Network guidelines. ${ }^{17} \mathrm{We}$ excluded the AML-M3 patients as their pathogeneses and treatments deviated significantly from those of other AML patients. A total of 27 patients diagnosed with iron anemia and idiopathic thrombocytopenic purpura were enrolled and formed the control group. The ratio of leukemic cells was identified through flow cytometric analysis. Bone marrow mononuclear cells (BMMCs) of the patient samples were prepared through Ficoll-Hypaque density gradient centrifugation.

\section{Cell lines and culture conditions}

Human K562 cells were kindly provided by the Tumor Research Institute of the Central South University. The use of these cells was approved by the ethics committee of the institutional review board of The Third Xiangya Hospital, Central South University (IRB2018-S090). Cells were cultured in RPMI 1640 medium containing 10\% fetal bovine serum in a $5 \%$ carbon dioxide incubator at $37{ }^{\circ} \mathrm{C}$ and harvested in the exponential growth phase.

\section{Adenoviral vectors}

The adenoviral vectors (control and shADAR1) were provided by Dr. Wang (Pittsburgh, PA, USA). The generation and purification of Ad-control and Ad-shADAR1, as well as the identification of TCID50, were performed by the Genechem Gene Technology Company Ltd. (Shanghai, China). Total RNA and protein were prepared $48 \mathrm{~h}$ posttransfection, and subjected to quantitative-polymerase chain reaction (qPCR), Western blotting, and in-vivo analysis.

\section{Isolation of total RNA and real-time PCR}

Total RNA from BMMCs and K562 cells were isolated using the TRIzol reagent according to the instructions provided by the manufacturer (Invitrogen Life Technologies, USA). cDNA was synthesized using Moloney Murine Leukemia Virus Reverse Transcriptase (Thermo Fisher Scientific, Waltham, MA, USA) following the recommendations provided by the manufacturer. The mRNA expression levels were measured via relative quantitative real-time PCR (qRT-PCR) using the CFX96 ${ }^{\mathrm{TM}}$ Real-Time System (Bio-Rad Laboratories, Hercules, CA, USA). The real-time PCR amplification was performed using the SYBR Green Mix (Bio-Rad Laboratories). All reactions were performed in triplicate. The expression levels were analyzed using the cycle quantification value calculated through the $\Delta$ Cycle threshold method. The amplification efficiencies were validated showing identical slopes. A negative control without template was also included. Human umbilical vein endothelial cells were used as a positive control. The following primers were used:

ADAR1-Forward: TGGCAGCCTCCGGGTG, ADAR1-Reverse: TGTCTGTGCTCATAGCCTTG; $\beta$-actin-Forward: CAGGGCGTGATGGTGGGCA, 
$\beta$-actin-Reverse: CAAACATCATCTGGGTCATCTTC $\mathrm{TC}$;

$\beta$-catenin-Forward: TGGTGACAGGGAAGACATCA, $\beta$-catenin-Reverse: CCATAGTGAAGGCGAACTGC; c-Myc-Forward: GGAGACACCGCCCACCA, c-Myc-Reverse: GCGCTGCGTAGTTGTGCTG; TCF4-Forward: GTACTGACCCAGTGCCATCTT, TCF4-Reverse:AGTTTGATGGCAAGGAAGGCT; CCND2-Forward:ACCACCTTCTTGGCTAAGTCC, CCND2-Reverse: TGCTGAACTTTGCACTCCCT.

\section{Western blotting}

The BMMCs, K562 cells, and mouse tumors were treated with radioimmunoprecipitation assay lysis buffer for $30 \mathrm{~min}$. After centrifugation at $12,000 \times$ rpm for $15 \mathrm{~min}$, the supernatant was used for the whole-protein lysate samples. Protein concentrations were determined using the bicinchoninic acid standard method. Protein extracts were analyzed through sodium dodecyl sulfate-polyacrylamide gel electrophoresis using $6-12 \%$ gels, followed by transfer to polyvinylidene fluoride membranes. Subsequently, the membranes were blocked in non-fat milk for approximately $1 \mathrm{~h}$ at room temperature, and subsequently incubated for $2 \mathrm{~h}$ at room temperature or overnight at $4{ }^{\circ} \mathrm{C}$ with primary antibodies. The membranes were incubated with anti-ADAR1 (1:200; Santa Cruz Biotechnology, Santa Cruz, CA, USA); anti- $\beta$ catenin (1:2,000; Proteintech, Wuhan, China); anti-c-Myc (1:1,000; Cell Signaling Technology, Danvers, MA, USA); anti-transcription factor 4 (anti-TCF4: 1:2,000; Proteintech); anti-Cyclin D2 (anti-CCND2: 1:2,000; Proteintech), or anti$\beta$-actin (1:5,000; Proteintech) antibodies at $4{ }^{\circ} \mathrm{C}$ overnight. This was followed by incubation with horseradish peroxidase-conjugated secondary antibodies (1:5,000; Proteintech). Immunoreactive bands were visualized using the enhanced chemiluminescence method according to the instructions provided by the manufacturer (Bio-Rad Laboratories). All Western blotting analyses were performed in triplicate independent experiments.

\section{Cell proliferation assay and colony formation in soft agar}

K562 cells were seeded in a 96-well plate at a density of $1 \times 10^{3}$ cells per well. The growth rate of cells was measured using the Cell Proliferation MTT Kit (SigmaAldrich, St. Louis, MO, USA) according to the instructions provided by the manufacturer. Three independent experiments were performed. For the soft agar assay,
$1 \times 10^{3}$ cells were seeded in $0.6 \%$ low-melting agarose (Sigma-Aldrich) and placed at the bottom of a six-well plate. After culture for 2 weeks, surviving colonies ( $>50$ cells per colony) were counted and stained with crystal violet (Sigma-Aldrich). Triplicate independent experiments were performed.

\section{Cell cycle analysis}

Cell cycle analysis was performed using flow cytometry as previously described. K562 cells, which were infected with Ad-control or Ad-shADAR1, were fixed in $70 \%$ ethanol for $24 \mathrm{~h}$. Subsequently, cells were washed thrice with pre-cooled phosphate-buffered saline (PBS) and incubated with RNase A $(0.2 \mathrm{mg} / \mathrm{mL})$ diluted in pre-cooled PBS. Propidium iodide was subsequently added, and the samples were analyzed with the FACScalibur Flow Cytometer (BD Biosciences, San Jose, CA, USA).

\section{In-vivo analysis}

Evaluation of the in-vivo efficacy of ADAR1 in K562 cells was performed using a xenograft model in immunodeficient nude mice. Male nude mice (aged 5-7 weeks) were housed under conditions of constant photoperiod (12-h light/dark cycles) with sterilized food and water. Prior to inoculation, K562 cells were washed twice with PBS and resuspended in RPMI-1640. Each mouse was subcutaneously inoculated over the flank with $1 \times 10^{7} \mathrm{~K} 562$ cells in a total volume of $0.2 \mathrm{~mL}$. Tumor diameter was measured once every 3 days using calipers, and tumor volume was calculated using a standard formula: width $^{2} \times$ length $\times 0.52$. Body weights of the mice were measured once every 3 days. The mice were sacrificed 40 days after inoculation through cervical dislocation. The tumor tissues were removed and stored at $-80^{\circ} \mathrm{C}$ until further analysis. The Animal Experiment Committee of the Central South University approved all the protocols for the care and treatment of animals. This research adhered to the instructive notions for the care for laboratory animals, released by the Ministry of Science and Technology (China) on September 30th, 2006.

\section{Immunohistochemical staining}

The tumor sections were deparaffinized, rehydrated, and washed with PBS. Antigen retrieval was performed in $10 \mathrm{mM}$ sodium citrate buffer at $\mathrm{pH} 6.0$ for $20 \mathrm{~min}$ in a microwave oven. After blocking with $3 \%$ hydrogen peroxide for $10 \mathrm{~min}$, the sections were incubated with $0.1 \%$ TritonX-100 in PBS for 20 min at room temperature, and subsequently immersed in a blocking solution of 5\% 
bovine serum albumin for $20 \mathrm{~min}$ at room temperature. Primary antibodies (1:100) were added in an Immunohistochemistry Antibody Diluent, and the sections were incubated overnight at $4{ }^{\circ} \mathrm{C}$. Subsequently, the sections were rinsed with PBS and incubated for $1 \mathrm{~h}$ at room temperature with a horseradish peroxidase-conjugated Goat anti-Rabbit IgG polyclonal antibody. The sections were rinsed with PBS and the signal was developed using the peroxidase substrate 3,3'-diaminobenzidine, which appears as a brown reaction product. All sections were counterstained with hematoxylin and images were captured under a microscope.

\section{Statistical analysis}

The SPSS version 21.0 software (IBM Corp., Armonk, NY, USA) was used to perform data analyses. The expression levels of ADAR1 in clinical samples were compared using the Wilcoxon signed-rank test. Spearman correlation coefficients were used to evaluate the positive correlation between the mRNA expression of ADAR1 and ratio of leukemic cells in clinical samples. Quantitative data are presented as mean \pm standard error of the mean. The chi-squared test was used to determine the significance of differences among the covariates. The significance of the in-vitro data was determined using the Student's $t$-test (two-tailed). A $P<0.05$ denoted statistically significant difference.

\section{Results}

Expression of ADARI is increased in AML patients and positively correlated with leukemic cells

As shown in Figure 1A, we first examined the mRNA expression of ADAR1 in BMMCs obtained from different AML patients and controls using the qPCR method. The results revealed that the expression of ADAR1 mRNA was significantly higher in the BMMCs of AML patients, whereas it was relatively lower in the AML-CR and control group. Spearman analysis was used to detect the link between the expression of ADAR1 mRNA and the ratio of leukemic cells. As shown in Figure 1B, we found that the levels of ADAR1 mRNA were positively correlated with the ratio of leukemic cells (Figure 1B: $\mathrm{r}=0.432 ; P<0.001$ ). Subsequently, we examined the expression of ADAR1 protein in different AML patients and controls using Western blotting. Owing to the limited volumes of clinical samples extracted from bone marrow, we were only able to examine 32 AML samples, 18 AML-CR samples, and 15 control samples. The results showed that the AML patients had the highest levels of ADAR1 protein, whereas AMLCR patients and controls did not demonstrate a positive expression of ADAR1 protein (Figure 1C and D).

\section{Silencing of ADARI contributes to the decrease in the proliferation of leukemic cells in vitro}

Considering that AML patients express relatively higher levels of ADAR1, we use adenovirus transfection to silence its expression in K562 cells. The transfection efficiency of shADAR1 in K562 cells was evaluated by qPCR and Western blotting (Figure 2A and B). As expected, after transfection, the level of ADAR1 in K562 cells was significantly decreased by approximately $90 \%$ compared with that measured in the Ad-control. Notably, the transfection efficiency was sustained for 3 weeks (data not shown). For functional analysis, cells transfected with shRNA targeting ADAR1 were found to be less tumorigenic, as manifested by the decreased rates in cell growth (Figure 2C) and the decreased capability for colony focus formation (Figure 2D and E). Additionally, a significant increase was detected in the population of cells at the $\mathrm{G} 0 / 1$ phase at day 5 , along with a decrease in cells at the G2 phase compared with control cells (Figure 2F and G).

\section{Silencing of ADARI in K562 cells results in the inhibition of wingless-int (wnt) pathway effectors}

We examined the expression levels of several signaling pathways, which play important roles in regulating the proliferation of AML, to identify the molecular mechanisms involved in the effects of ADAR1 on the proliferation of AML cells. As shown in Figure 3A, we found that several Wnt pathway effectors ( $\beta$-catenin, c-Myc, TCF4, and CCND2) were downregulated in shADAR1 cells compared with control cells. This result was further confirmed by Western blotting in shADAR1 and control cells (Figure 3B). Our results indicated that ADAR1 may regulate the proliferation of AML cells through the Wnt pathway.

\section{Silencing of ADARI inhibits the proliferation of AML cells and wnt pathway in vivo}

Immunodeficient nude mice were subcutaneously injected with K562 cells transduced with Ad-Control or AdshADAR1 to further investigate the effect of ADAR1 on 

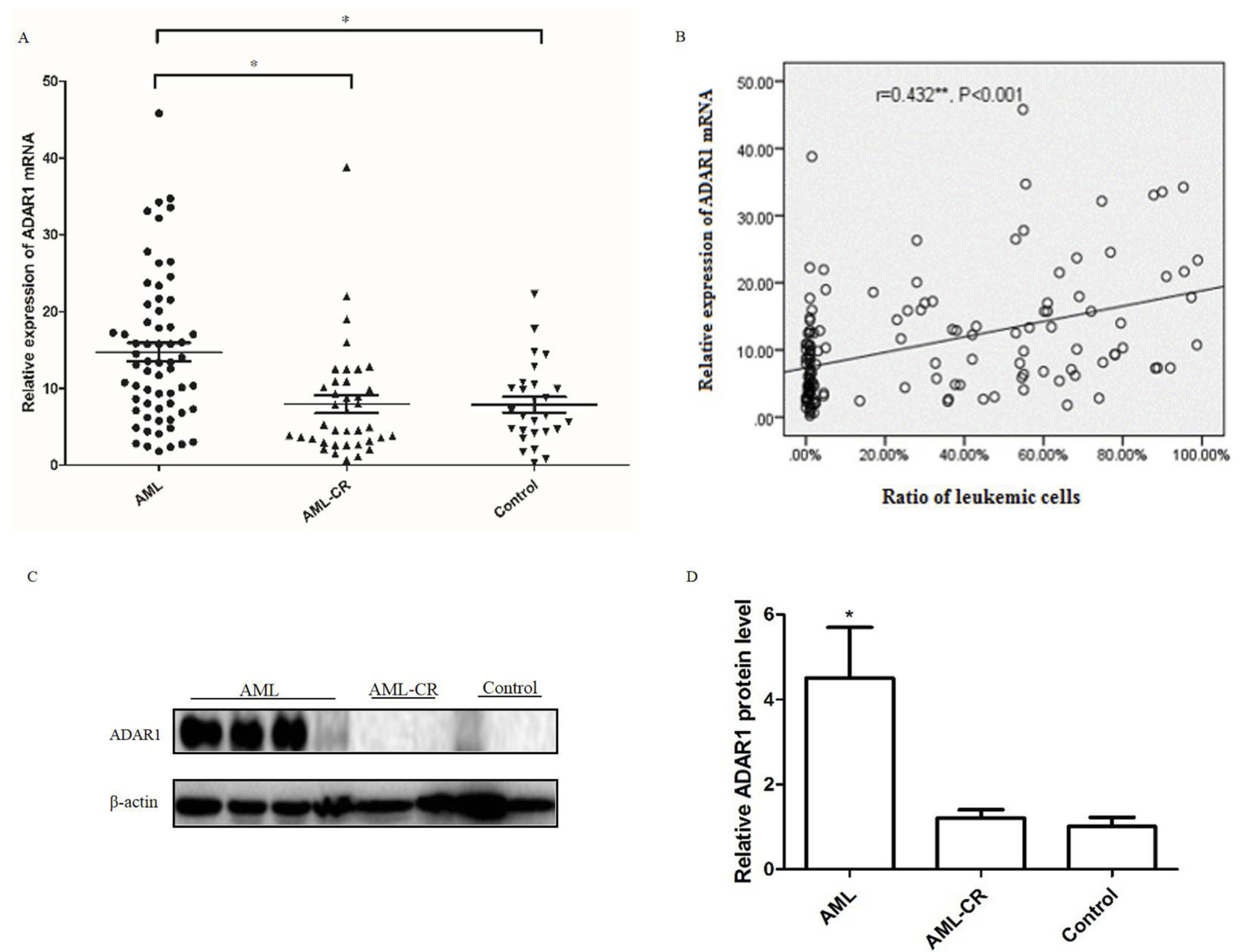

Figure I Expressions of ADARI in AML patients and the control subjects. (A) The mRNA levels of ADARI in AML patients and control subjects were measured by qPCR (B) The relationship between the levels of ADARI mRNA and the ratio of leukemic cells. (C) Representative pictures of three independent experiments that gave similar results were shown. (D) Summarized data of Western blots of the levels of ADARI protein in AML patient and control group. Data were shown as mean \pm S.E.M. $* P<0.05$. $*^{*} r=0.432, P<0.001$ Spearman correlation analysis.

the proliferation of AML cells in vivo. Six days after injection, all mice had developed detectable tumors. On day 14 after tumor inoculation, the mice harboring tumors with reduced expression of ADAR1 showed drastically decreased tumor growth (Figure 4A) compared with the control group, as demonstrated by their substantially reduced body weight (Figure 4B). The tumors were resected and analyzed as follows. Using Western blotting and immunohistochemical staining, it was shown that tumor tissues derived from shADAR1-transduced cells exhibited reduced expression of $\beta$-catenin, c-Myc, TCF4, and CCND2 compared with the control group (Figure $4 \mathrm{C}-\mathrm{E}$ ). These results suggested that downregulation of ADAR1 may have inhibited the proliferation of AML cells via the Wnt pathway.

\section{Discussion}

Markedly high expression levels of ADAR1 were previously reported in Chinese pediatric patients with leukemia. ${ }^{16} \mathrm{~A}$ study utilizing a chronic myeloid leukemia mouse model showed that deletion of ADAR1 may normalize the peripheral white blood count and eliminate leukemic cells, revealing a new role of ADAR1 in myeloid leukemia cells. ${ }^{18}$ These previous findings prompt us to investigate the role of ADAR1 in the development of adult AML. In the present study, we found that the expression of ADAR1 was significantly higher in patients with AML, and silencing of ADAR1 decreased the proliferation of AML cells and expression of Wnt effectors in vitro or in vivo. Our data indicated that ADAR1 may promote the proliferation of AML cells by regulating the Wnt signaling pathway.

Previous reports have shown that ADAR1 is involved in regulating the proliferation, apoptosis, and invasion of numerous types of cancer, including gastric, ${ }^{19}$ hepatocellular carcinoma, ${ }^{11}$ and lung cancer. ${ }^{12}$ However, there is limited information regarding the expression levels and functions of ADAR1 in adult AML. Our findings demonstrated that the levels of ADAR1 mRNA and protein were markedly higher in patients with AML compared with 


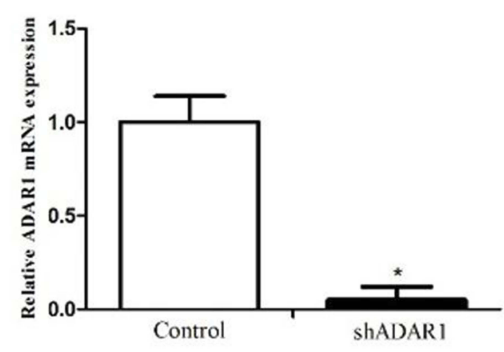

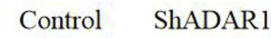

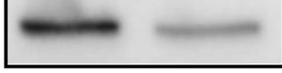

$\beta$-actin

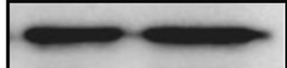

C

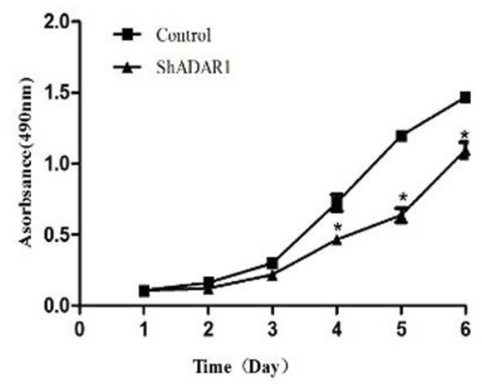

D

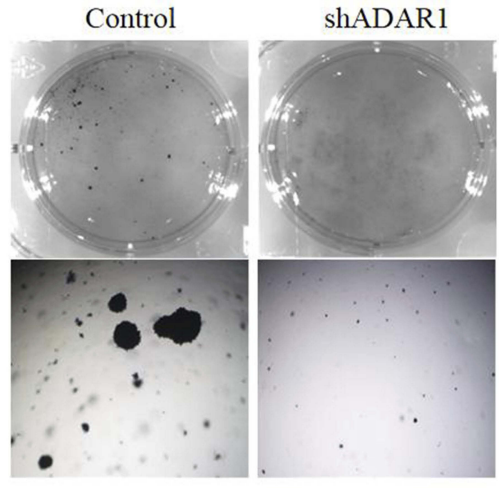

$\mathrm{E}$

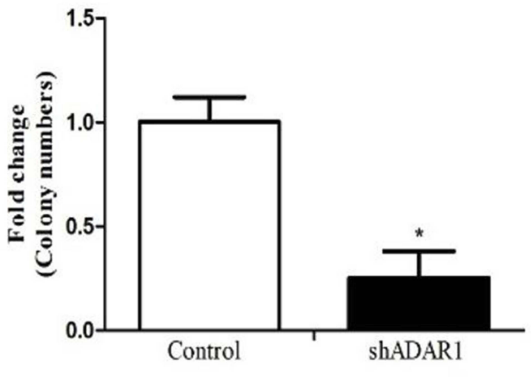

$\mathrm{F}$

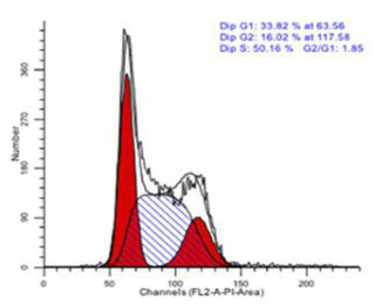

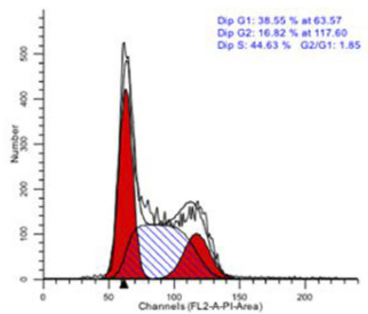

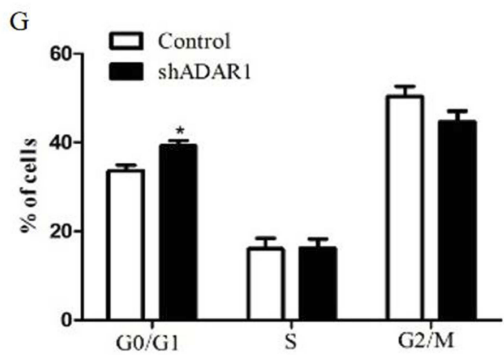

Figure 2 Silence of ADARI in AML cell line inhibits the proliferation of K562 cells. (A) The transfection efficiency of adenoviral vectors were measured by qPCR. (B) The transfection efficiency of adenoviral vectors were measured by Western Blot. (C) The effects of ADARI knockdown on cell proliferation in K562 cells measured by MTT assay. (D) The effects of ADARI knockdown on colony formation capacity of K562 cells. The same amounts of related cells were plated into a 6-well plate. Cell colonies were stained and counted after two weeks. (E) Summarized data of the colony formation assay. (F) The effects of ADARI knockdown on cell cycle of K562 cells measured by flow-cytometry assay. (G) Summarized data of the cell cycle analysis. All the above experiments were repeated at least three times. The data represents mean \pm SD of three independent experiments. $* P<0.05$.

those measured in the AML-CR or control groups. Moreover, the ratio of malignant leukemic cells showed a positive correlation with the expression of ADAR1 mRNA ( $\mathrm{r}=0.426 ; P<0.001)$. Of note, patients with AML showed a positive expression of ADAR1 protein, whereas the AML-CR and controls did not express the ADAR1 protein. This observation suggested higher specificity in the level of ADAR1 protein in AML samples. Based on these results, we propose that ADAR1 is highly expressed in AML patients and may play an oncogenic role in the 

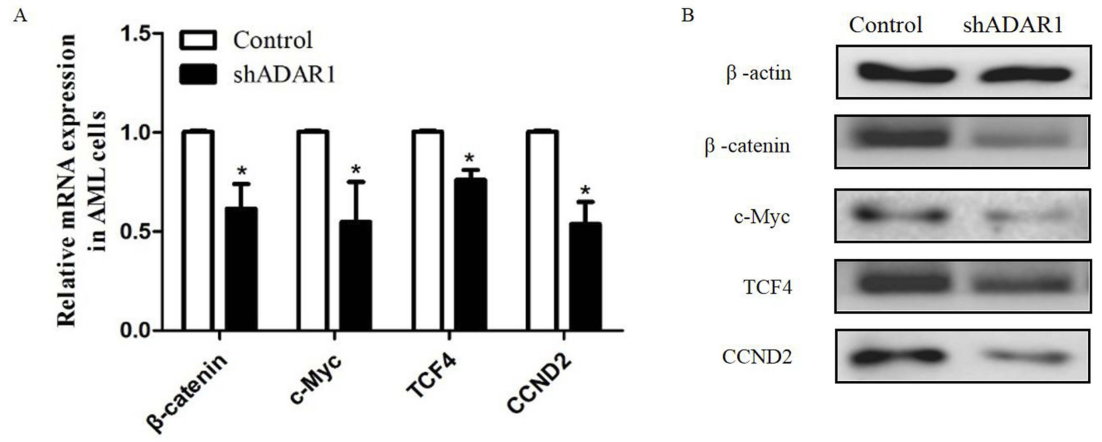

Figure 3 Silence of ADARI inhibits the expressions of Wnt effectors. (A) The effect of ADARI knockdown on the expressions of Wnt effectors measured by qPCR. (B) The effect of ADARI knockdown on the expressions of Wnt effectors measured by Western Blot. All the above experiments were repeated at least three times. The data represents mean \pm SD of three independent experiments. $* P<0.05$.

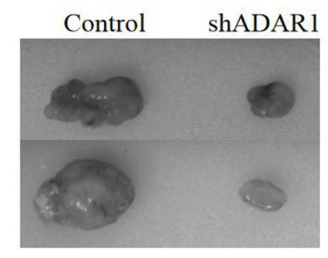

C

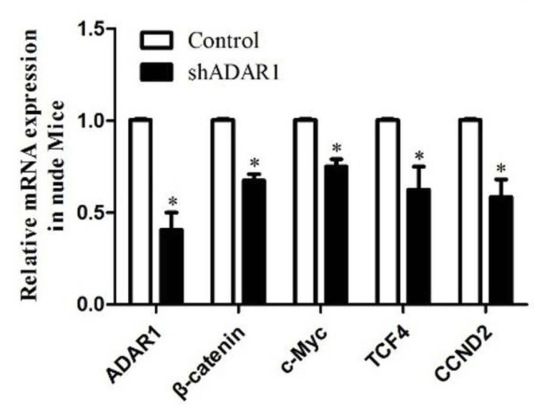

E

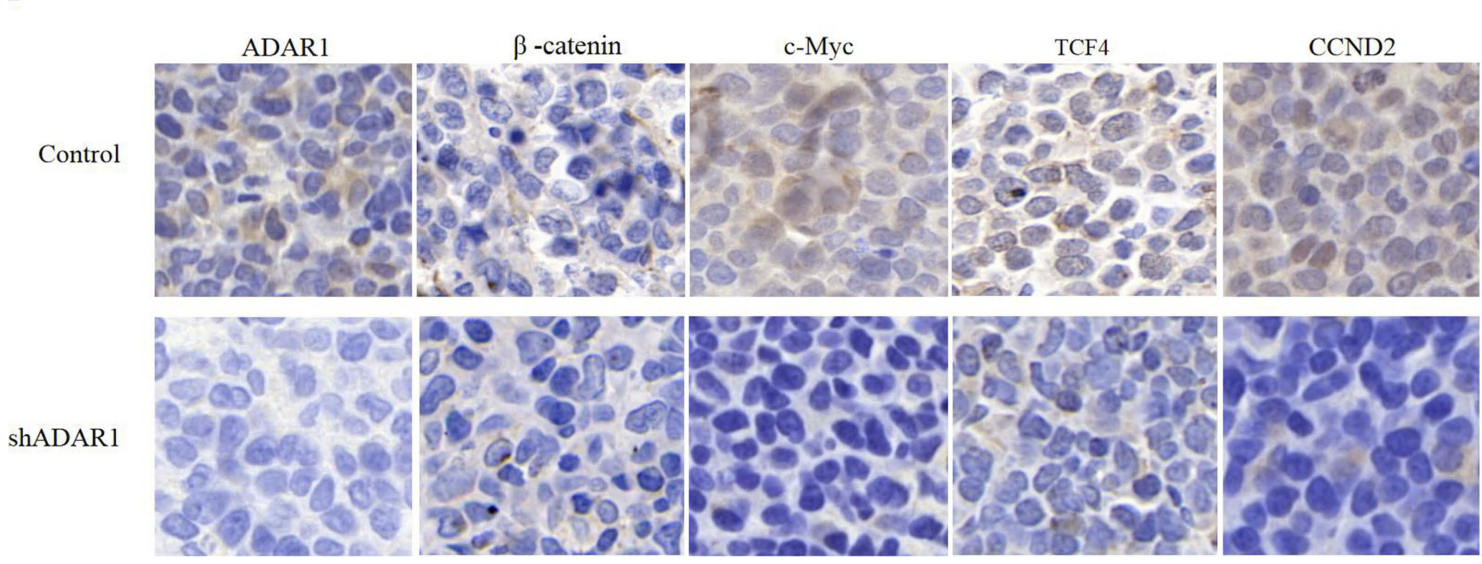

Figure 4 Silence the ADARI inhibits AML proliferation and Wnt pathway in vivo. (A) The effects of ADARI knockdown on cell proliferation in K562 cells in vivo. (B) Summarized data of the tumor weight. (C) The effect of ADARI knockdown on the expressions of Wnt effectors measured by qPCR in vivo. (D) The effect of ADARI knockdown on the expressions of Wnt effectors measured by Western Blot in vivo. (E) The effect of ADARI knockdown on the expressions of Wnt effectors measured by Immunohistochemical staining. All the above experiments were repeated at least three times. The data represents mean \pm SD of three independent experiments. $* P<0.05$. 
development of AML. However, further flow sorting of AML cells with specific markers in BMMCs may help to precisely investigate the expression levels of ADAR1.

The role of ADAR1 in the development of hematopoiesis is well established. ${ }^{10}$ Thus, ADAR1 is essential for the survival of hematopoietic cells. In addition, ADAR1 is important in the development of malignant leukemic cells. ${ }^{15}$ Considering that ADAR 1 is highly expressed in patients with AML, we downregulated the expression of ADAR1 using adenoviral infections for a detailed investigation of its functions. Our functional assays demonstrated that depletion of ADAR1 may significantly inhibit the proliferation and clonal formation of leukemic cells in vitro and in vivo. Moreover, we showed that the downregulation of ADAR1 significantly induced cell cycle arrest at G0/1, which is consistent with the reduced expression of CCND2. These data indicated that ADAR1 is involved in modulating the proliferation of AML cells by affecting the leukemic cell cycle. Similar effects of ADAR1 on the proliferation of tumor cells were also observed in other types of cancer. ${ }^{12,14,20}$ The results of this study provide a novel understanding of the role of ADAR1 in the development of adult AML cells. However, the specific mechanisms through which ADAR1 regulates changes in the leukemic cell cycle and proliferation remain unknown.

There are numerous signaling pathways (mTOR, nuclear factor- $\kappa \mathrm{B}$, NOTCH, and $\mathrm{Wnt}$ ) participating in the regulation of proliferation and the cell cycle of AML cells. ${ }^{21-24}$ The mTOR and Wnt pathways have been previously reported to modulate the functions of ADAR1. ${ }^{19,25}$ It has been shown that loss of ADAR1 may alter the expression of Wnt targets, including CCND1 and c-Myc, in intestinal functions. ${ }^{25}$ The canonical Wnt signaling pathway is activated by the entry of $\beta$-catenin into the nucleus, and promotes transcriptional activation in conjunction with TCF4. Furthermore, it activates target genes that are related to the survival and proliferation of cancer cells. ${ }^{26}$ We recognized that the CCND2 is one of the important target genes of the canonical Wnt pathway. Therefore, we examined other key regulators of the Wnt pathway ( $\beta$-catenin, c-Myc, TCF4) to further investigate the role of this pathway after knockdown of ADAR1. We found that deletion of ADAR1 in AML cells resulted in decreased expression of these Wnt target genes. Moreover, the in-vivo results also confirmed that the Wnt signaling pathway may be the downstream target of ADAR1 in modulating the proliferation of AML cells. These data imply the presence of a novel mechanism involved in the effects of ADAR1 on the proliferation and cell cycle of AML cells. However, whether the regulation of AML cell proliferation by ADAR1 is merely dependent on the Wnt pathway warrants further investigation.

\section{Conclusion}

Our study reported that ADAR1 may function as an oncogene in adult AML. The mRNA expression of ADAR1 was associated with the ratio of leukemic cells. In addition, ADAR1 displayed growth-enhancing activity in vitro and in vivo, as demonstrated by the depletion experiments in cell culture and mouse models. In the analysis of the mechanism, deletion of ADAR1 resulted in decreased expression of certain target genes involved in the Wnt pathway. These findings provide new insights into the biological value of ADAR1 in the proliferation of AML cells, and suggest that ADAR1 may be useful in the detection of additional biomarkers related to the development of AML.

\section{Acknowledgments}

This paper was presented at The American Society of Hematology 59th Annual Meeting, 2017, as a poster presentation with interim findings. The poster's abstract was published in "Poster Abstracts" in Blood and is available from: http:// www.bloodjournal.org/content/130/Suppl_1/2513. This work was supported by the National Natural Science Foundation of China (grant nos. 81670203 and 81870166, for Jing Liu and grant no. 81870165 for Xin Li).

\section{Disclosure}

The authors report no conflicts of interest in this work.

\section{References}

1. Gupta V, Tallman MS, Weisdorf DJ. Allogeneic hematopoietic cell transplantation for adults with acute myeloid leukemia: myths, controversies, and unknowns. Blood. 2011;117(8):2307-2318. doi:10.1182/blood-2010-10-265603

2. Khaled S, Al MM, Marcucci G. Acute myeloid leukemia: biologic, prognostic, and therapeutic insights. Oncology (Williston Park). 2016;30(4):318-329.

3. Hatfield KJ, Reikvam H, Bruserud Ø. Identification of a subset of patients with acute myeloid leukemia characterized by long-termin vitro proliferation and altered cell cycle regulation of the leukemic cells. Expert Opin Ther Tar. 2014;18(11):1237-1251. doi:10.1517/ 14728222.2014.957671

4. Feldman EJ. Novel therapeutics for therapy-related acute myeloid leukemia: 2014. Clin Lymphoma Myeloma Leuk. 2015;15:S91-S93. doi:10.1016/j.clml.2015.02.031

5. Eriksson A, Lennartsson A, Lehmann S. Epigenetic aberrations in acute myeloid leukemia: early key events during leukemogenesis. Exp Hematol. 2015;43(8):609-624. doi:10.1016/j.exphem.2015.05. 009 
6. Sanchez M, Levine RL, Rampal R. Integrating genomics into prognostic models for AML. Semin Hematol. 2014;51(4):298-305. doi:10.1053/j.seminhematol.2014.08.002

7. Wang Q, Khillan J, Gadue P, Nishikura K. Requirement of the RNA editing deaminase ADAR1 gene for embryonic erythropoiesis. Science. 2000;290(5497):1765-1768. doi:10.1126/science.290.5497.1765

8. Cachat A, Alais S, Chevalier SA, et al. ADAR1 enhances HTLV-1 and HTLV-2 replication through inhibition of PKR activity. Retrovirology. 2014;11:93. doi:10.1186/s12977-014-0093-9

9. Qiao JJ, Chan TH, Qin YR, Chen L. ADAR1: a promising new biomarker for esophageal squamous cell carcinoma? Expert Rev Anticancer Ther. 2014;14(8):865-868. doi:10.1586/14737140.2014.928595

10. Hartner JC, Walkley CR, Lu J, Orkin SH. ADAR1 is essential for the maintenance of hematopoiesis and suppression of interferon signaling. Nat Immunol. 2009;10(1):109-115. doi:10.1038/ni.1680

11. Chan THM, Lin $\mathrm{CH}$, Qi L, et al. A disrupted RNA editing balance mediated by ADARs (Adenosine DeAminases that act on RNA) in human hepatocellular carcinoma. Gut. 2014;63(5):832-843. doi:10.1 136/gutjnl-2012-304037

12. Anadon C, Guil S, Simo-Riudalbas L, et al. Gene amplificationassociated overexpression of the RNA editing enzyme ADAR1 enhances human lung tumorigenesis. Oncogene. 2016;35(33):4422. doi:10.1038/onc.2016.27

13. Chen Y, Wang H, Lin W, Shuai P. ADAR1 overexpression is associated with cervical cancer progression and angiogenesis. Diagn Pathol. 2017;12(1):12. doi:10.1186/s13000-017-0600-0

14. Nemlich Y, Greenberg E, Ortenberg R, et al. MicroRNA-mediated loss of ADAR1 in metastatic melanoma promotes tumor growth. $J$ Clin Invest. 2013;123(6):2703-2718. doi:10.1172/JCI62980

15. Rossetti C, Picardi E, Ye M, et al. RNA editing signature during myeloid leukemia cell differentiation. Leukemia. 2017;31(12):2824 2832. doi:10.1038/leu.2017.134

16. Ma CH, Chong JH, Guo Y, et al. Abnormal expression of ADAR1 isoforms in Chinese pediatric acute leukemias. Biochem Biophys Res Commun. 2011;406(2):245-251. doi:10.1016/j.bbrc.2011.02.025
17. National comprehensive cancer network: NCCN categories of evidence and consensus., 2018.

18. Steinman RA, Yang Q, Gasparetto M, et al. Deletion of the RNAediting enzyme ADAR1 causes regression of established chronic myelogenous leukemia in mice. Int J Cancer. 2013;132(8):17411750. doi:10.1002/ijc. 27851

19. Dou N, Yu S, Ye X, Yang D, Li Y, Gao Y. Aberrant overexpression of ADAR1 promotes gastric cancer progression by activating mTOR/ p70S6K signaling. Oncotarget. 2016;7(52):86161-86173. doi:10.186 32/oncotarget.13354

20. Qin YR, Qiao JJ, Chan THM, et al. Adenosine-to-inosine RNA editing mediated by ADARs in esophageal squamous cell carcinoma. Cancer Res. 2014;74(3):840-851. doi:10.1158/0008-5472.CAN-132545

21. Gu Y, Masiero M, Banham AH. Notch signaling: its roles and therapeutic potential in hematological malignancies. Oncotarget. 2016;7(20):29804-29823. doi:10.18632/oncotarget.7772

22. Zhou J, Ching YQ, Chng WJ. Aberrant nuclear factor-kappa B activity in acute myeloid leukemia: from molecular pathogenesis to therapeutic target. Oncotarget. 2015;6(8):5490-5500. doi:10.18632/ oncotarget. 3545

23. Carneiro BA, Kaplan JB, Altman JK, Giles FJ, Platanias LC. Targeting mTOR signaling pathways and related negative feedback loops for the treatment of acute myeloid leukemia. Cancer Biol Ther. 2015;16(5):648-656. doi:10.4161/15384047.2014.987548

24. Mikesch JH, Steffen B, Berdel WE, Serve H, Muller-Tidow C. The emerging role of Wnt signaling in the pathogenesis of acute myeloid leukemia. Leukemia. 2007;21(8):1638-1647. doi:10.1038/sj.leu.240 4732

25. Qiu W, Wang X, Buchanan M, et al. ADAR1 is essential for intestinal homeostasis and stem cell maintenance. Cell Death Dis. 2013;4(4): e599. doi:10.1038/cddis.2013.125

26. Ge X, Wang X. Role of Wnt canonical pathway in hematological malignancies. J Hematol Oncol. 2010;3:33. doi:10.1186/1756-87223-50
Cancer Management and Research

\section{Publish your work in this journal}

Cancer Management and Research is an international, peer-reviewed open access journal focusing on cancer research and the optimal use of preventative and integrated treatment interventions to achieve improved outcomes, enhanced survival and quality of life for the cancer patient.
The manuscript management system is completely online and includes a very quick and fair peer-review system, which is all easy to use. Visit http://www.dovepress.com/testimonials.php to read real quotes from published authors. 